\title{
Importance of VIIIa Inactivation in a Mathematical Model for the Formation, Growth, and Lysis of Clots
}

\author{
P.P. Naidu, M. Anand * \\ Department of Chemical Engineering, Indian Institute of Technology Hyderabad \\ Yeddumailaram, Andhra Pradesh - 502205, India
}

\begin{abstract}
We perform a sensitivity analysis for a thus far unstudied mathematical model for the formation, growth and lysis of clots in vitro. The sensitivity analysis procedure uses an ensemble standard deviation for species concentrations, and is equivalent to a variance decomposition procedure also available in the literature. Our analysis shows that fibrin production is most sensitive to the rate constant governing activation of prothrombin to thrombin. Further, the time-averaged sum of all species' concentrations is most sensitive to the rate constants governing the inactivation of VIIIa (intrinsic as well as by APC). We therefore conclude that the rate constants for VIIIa inactivation affect the model the greatest: this conclusion must be experimentally verified to determine if such is indeed the case for hemostasis.
\end{abstract}

Keywords and phrases: VIIIa inactivation, mathematical model, sensitivity analysis, hemostasis, rate constant

Mathematics Subject Classification: 35K57, 49Q12, 92C40

\section{Introduction}

The ability of blood to form a self-sealing clot when a blood vessel is injured is crucial to healing. Without coagulation, even a minor cut or puncture wound would continue to bleed and lead to death. A deficiency in some of the protein factors involved in coagulation can result in hemorrhages following injury: one such deficiency leads to Hemophilia. Hemophilia is an inherited coagulation disorder characterized by the blood's inability to clot leading to prolonged bleeding even from the most minor of cuts. About $80 \%$ of hemophiliacs have type A [24] which is the result of deficiency of clotting factor VIII. Hemophilia type $\mathrm{B}$, on the other hand, is the result of deficiency of clotting factor IX. It is critical to know the sensitivity of clot formation, growth, and lysis to changes in pro-coagulant concentrations (e.g. of f-VIII/f-IX) as well as to changes in the rate constants governing their reactions (e.g. the Inactivation of f-VIIIa/f-IXa).

A mathematical model of hemostatic system function provides a theoretical means to test the effect of various coagulation factor deficiencies/excesses and enzymatic rate constant variations on hemostatic system function. Mathematical models for coagulation have increased in detail and scope since the mid1960s [15]: the more recent ones are quite detailed in their treatment of either segments of the coagulation cascade like the extrinsic pathway [10], or the extrinsic and intrinsic pathway [22], or the entire cascade

${ }^{*}$ Corresponding author. E-mail: anandm@iith.ac.in

(C) EDP Sciences, 2014 
along with fibrinolysis [2].The model in [2] is a complete, though by no means comprehensive, model for the biochemical reactions underlying the extrinsic coagulation pathway and fibrinolysis. This model has thus far not been studied extensively unlike other models for coagulation notably the ones in [13], [3], [10], [5]. The only studies available for this model are an extension to include the intrinsic pathway [14], and a stability analysis in [23]. We therefore select this particular model for study to understand it better.

We perform a sensitivity analysis of the model in [2] using the procedure in [8] so as to find the most sensitive rate constants of the model. The results will identify the rate constants that affect the model predictions for individual/all species the most/least, and will provide insights for possible experimental studies. The paper is organized as follows: We formulate the problem in the next section (Section 2). We then list the model equations to be analyzed, and also review the sensitivity analysis procedure in Section 3. In Section 4 results from the sensitivity analysis of the model are presented. The results are discussed in Section 5 and conclusions are made about the sensitivity of the model predictions to the individual rate constants.

\section{Problem formulation}

The phenomenon that we model is clot formation, growth, and lysis in quiescent plasma over a thrombogenic patch (of sub-endothelial proteins) circumscribed by a patch of endothelial cells. Platelet activation is initiated, and proceeds along with the extrinsic pathway of coagulation which is also initiated, when blood contacts the sub-endothelial proteins. Platelet activation leads to the formation of platelet aggregates whereas the extrinsic pathway of coagulation leads to the formation of thrombin, and thence, fibrin. The extrinsic, or tissue factor pathway, is one of the two coagulation pathways leading to the formation of thrombin; the second pathway is the intrinsic, or contact pathway. Both pathways consist of a series of proteolytic reactions involving the step-wise activation of coagulation factors. Each pathway is initiated by different factors, but both converge to a single common pathway that starts with the activation of coagulation factor X and leads to the conversion of prothrombin(factor II) to active thrombin (factor IIa). Thrombin converts fibrinogen (factor I) to Fibrin (factor Ia) monomers that polymerize into strands. In addition, thrombin activates coagulation factor XIII, which stabilizes fibrin and promotes its cross-linking to form a fibrin network. The fibrin strands bind with the (already formed) platelet aggregates to form a clot. The clot covers the thrombogenic patch and prevents it from contacting blood. It also interacts with the surrounding patch of endothelial cells to initiate fibrinolysis- the series of reactions that results in degradation of fibrin fibers- and thus clot lysis. This broad picture is host to a series of enzymatic reactions (with positive and negative feedback loops) that allow precise control of the process (see [9], [7]). To summarize: clot formation, growth, and lysis occurring in response to blood contacting a thrombogenic surface proceeds in three stages: coagulation, clot consolidation, and fibrinolysis. Each stage involves the extra-vascular compartment (immediately above the thrombogenic patch, and below the clot surface), the clot surface, and the intra-vascular compartment (above the endothelial cells) [6],[20], [19].

The biochemical reactions involved in the above process are found in [9], [7], and [20]: a select set of these constituents are chosen in [2] to represent the entire phenomenon from the extrinsic pathway of coagulation through fibrinolysis. A set of biochemical reactions involving these constituents is then used to represent the sequence of events occurring in the extrinsic pathway, the common pathway, the formation of fibrin, and the generation of plasmin. This list of reactions is complete but not comprehensive.

We select a total of 25 constituents: 23 constituents governed by equations plus the 2 membrane-bound enzyme complexes. A set of 23 coupled reaction-diffusion equations (for 23 constituents) are formulated to govern the generation and/or depletion of plasma enzymes and zymogens (XIa/XI, IXa/IX, Xa/X, IIa/II, Va/V, VIIIa/VIII, Plasmin/Plasminogen (PLA/PLS)), membrane bound enzyme complexes (IXaVIIIa and $\mathrm{Xa}-\mathrm{Va}$ ), regulatory proteins (activated protein $\mathrm{C} /$ protein $\mathrm{C}(\mathrm{APC} / \mathrm{PC})$ ), inhibitors (ATIII, TFPI, $\alpha_{1}$-antitrypsin $\left.\left(\alpha_{1} \mathrm{AT}\right), \alpha_{2} \mathrm{AP}\right)$, tissue plasminogen activator (tPA) and fibrin/fibrinogen (Ia/I). A schematic of these reactions is given in Figure 1, and these reactions are also listed in Tables 1 (Zymogen activation) and 2 (Enzyme inactivation). We note in particular that the constants governing the activation 
of Protein $\mathrm{C}$ by thrombin (Reaction (9a)) are in the presence of saturating concentrations of dissolved thrombomodulin. This is because activation of Protein $\mathrm{C}$ in the absence of thrombomodulin is negligibly slow compared to that in the presence of thrombomodulin. We introduce a numbering scheme for the reaction constants in Tables 1 and 2: we refer to this numbering scheme while presenting the results.

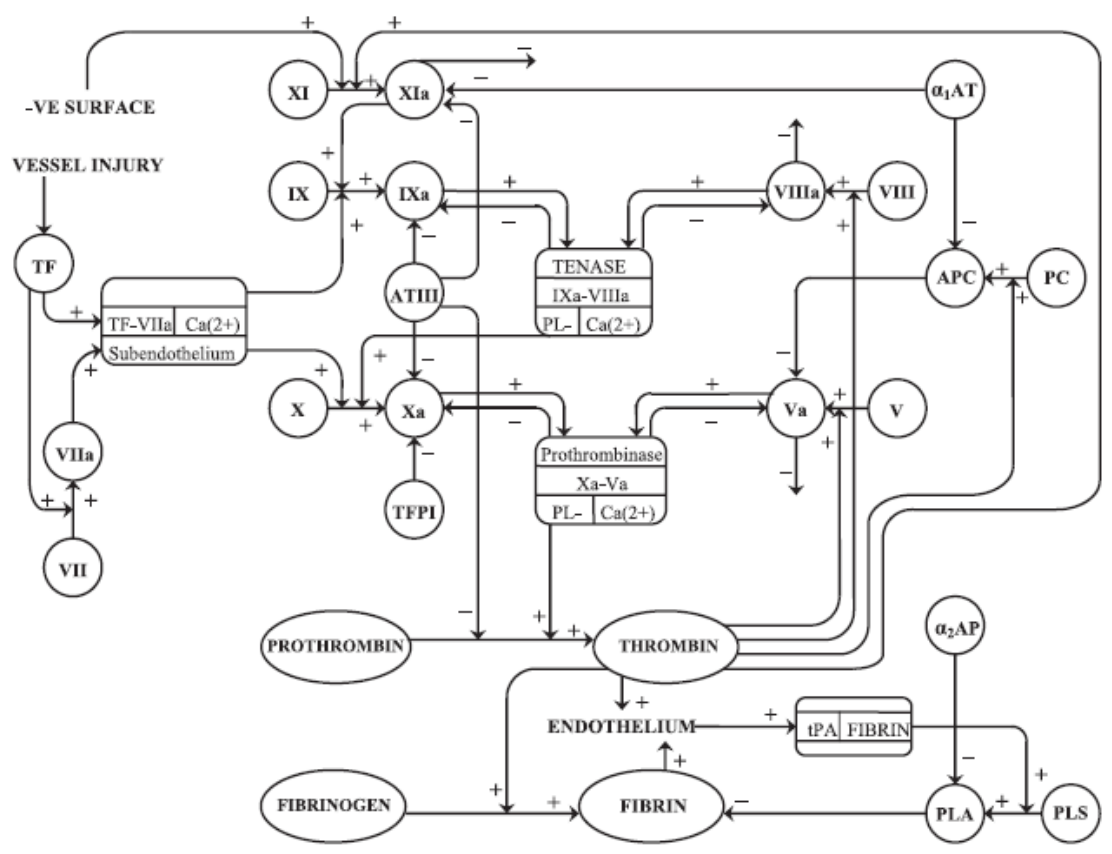

FIgURE 1. Biochemical model of selected reactions involved in the coagulation pathways and fibrinolysis. Plus sign indicates enzymatic activation. Minus signs indicate enzymatic inactivation or inhibition. Reprinted from [2] with permission from Elsevier.

Table 1: List of enzymatic reactions (Zymogen Activation).

\begin{tabular}{|c|c|c|}
\hline Rxn. No. & Rate constants (number) & Reaction \\
\hline (1a) & $k_{11}(1), K_{11 M}(2)$ & $\mathrm{XI} \stackrel{\mathrm{IIa}}{\longrightarrow} \mathrm{XIa}$ \\
\hline$(2 a)$ & $k_{9}(5), K_{9 M}(6)$ & $\mathrm{IX} \stackrel{\mathrm{XIa}}{\longrightarrow} \mathrm{IXa}$ \\
\hline (6a) & $k_{10}(8), K_{10 M}(9)$ & $\mathrm{X} \stackrel{Z}{\longrightarrow} \mathrm{Xa}$ \\
\hline$(8 \mathrm{a})$ & $k_{2}(11), K_{2 m}(12)$ & $\mathrm{II} \stackrel{W}{\longrightarrow} \mathrm{IIa}$ \\
\hline$(3 \mathrm{a})$ & $k_{8}(14), K_{8 M}(15)$ & VIII $\stackrel{\text { IIa }}{\longrightarrow}$ VIIIa \\
\hline$(4 a)$ & $K_{d Z}(19)$ & $\mathrm{VIIIa}+\mathrm{IXa} \stackrel{\mathrm{PL}^{-}}{\rightleftharpoons} \mathrm{Z}$ \\
\hline (5a) & $k_{5}(20), K_{5 M}(21)$ & $\mathrm{V} \stackrel{\mathrm{IIa}}{\longrightarrow} \mathrm{Va}$ \\
\hline$(7 a)$ & $K_{d W}(25)$ & $\mathrm{Va}+\mathrm{Xa} \stackrel{\mathrm{PL}^{-}}{\rightleftharpoons} \mathrm{W}$ \\
\hline$(9 a)$ & $k_{P C}(26), K_{P C M}(27)$ & $\mathrm{PC} \stackrel{\mathrm{IIa}}{\longrightarrow} \mathrm{APC}$ \\
\hline$(10 \mathrm{a})$ & $k_{1}(30), K_{1 M}(31)$ & $\mathrm{I} \stackrel{\mathrm{IIa}}{\longrightarrow} \mathrm{Ia}$ \\
\hline$(11 \mathrm{a})$ & $k_{P L A}(34), K_{P L A M}(35)$ & $\mathrm{PLS}^{t P A-I a} \stackrel{\mathrm{PLA}}{\longrightarrow}$ \\
\hline
\end{tabular}


Table 2: List of enzymatic reactions (Enzyme Inactivation).

\begin{tabular}{|c|c|c|}
\hline Rxn. No. & Rate constants (number) & Reaction \\
\hline (1b) & $h_{11}^{A 3}(3)$ & $\mathrm{XIa}+$ ATIII $\rightarrow$ XIa-ATIII \\
\hline (1c) & $h_{11}^{L 1}(4)$ & $\mathrm{XIa}+\alpha_{1} \mathrm{AT} \rightarrow \mathrm{XIa}-\alpha_{1} \mathrm{AT}$ \\
\hline$(2 b)$ & $h_{9}(7)$ & IXa + ATIII $\rightarrow$ IXa-ATIII \\
\hline$(6 \mathrm{~b})$ & $h_{10}(10)$ & $\mathrm{Xa}+\mathrm{ATIII} \rightarrow \mathrm{Xa}$-ATIII \\
\hline$(6 c)$ & $h_{T F P I}(29)$ & $\mathrm{Xa}+\mathrm{TFPI} \rightarrow \mathrm{Xa-TFPI}$ \\
\hline (8b) & $h_{2}(13)$ & IIa + ATIII $\rightarrow$ IIa-ATIII \\
\hline$(3 \mathrm{~b})$ & $h_{8}(16)$ & VIIIa $\rightarrow$ VIII $_{i}$ \\
\hline (3c) & $h_{C 8}(17), H_{C 8 M}(18)$ & VIIIa $\stackrel{A P C}{\rightarrow}$ VIII $_{i}$ \\
\hline$(5 \mathrm{~b})$ & $h_{5}(22)$ & $\mathrm{Va} \rightarrow \mathrm{V}_{i}$ \\
\hline$(5 c)$ & $h_{C 5}(23), H_{C 5 M}(24)$ & $\mathrm{Va}^{A P C} \rightarrow \mathrm{V}_{i}$ \\
\hline$(9 b)$ & $h_{P C}(28)$ & $\mathrm{APC}+\alpha_{1} \mathrm{AT} \rightarrow \mathrm{APC}-\alpha_{1} \mathrm{AT}$ \\
\hline$(10 \mathrm{~b})$ & $h_{1}(32), H_{1 M}(33)$ & $\mathrm{Ia}+\mathrm{PLA} \rightarrow \mathrm{I}_{i}$ \\
\hline$(11 b)$ & $h_{P L A}(36)$ & $\mathrm{PLA}+\alpha_{2} \mathrm{AP} \rightarrow \mathrm{PLA}-\alpha_{2} \mathrm{AP}$ \\
\hline
\end{tabular}

The response of the model equations depends on the i) flux boundary conditions, which regulate the threshold response of the system and the concentrations of the reactions within the domain, and ii) the initial conditions consisting of the initial concentrations of the reactants. The flux boundary conditions represent the surface stimulus for the reactions: the size of the thrombogenic patch (as reflected in the concentration of surface bound TF-VIIa complex), and the extent of endothelial cell activity in secreting tPA (either constitutively, or upon action by thrombin and fibrin). The initial concentrations of the reactants are set to be those in human plasma for the zymogens, whereas, a $0.1 \%$ initial level of activation is assumed for the activated enzymes. Further, the model specifies clot formation, growth, and lysis as occurring in the following manner:

- Clot formation is defined to occur when fibrin concentration equals or exceeds a specific concentration $C L_{c r}$ at a point in the domain;

- Clot growth is determined by tracking, in time, the extent of the spatial domain where concentration of fibrin is $\geq C L_{c r}$;

- Clot lysis occurs when fibrinolysis progresses such that the fibrin concentration becomes $<C L_{c r}$ after previously equaling or exceeding it.

Sensitivity analysis for the above model will identify those parameters (rate constants) to which the predictions of the model (reactant concentrations) are most/least sensitive. This involves varying each rate constant in the model in a predesignated manner, and obtaining the resultant variation in each species' concentration. In the literature surveyed, we found two sensitivity analysis procedures for coagulation models: the one in [8], and the other in [17]. The former is a sampling based method using an ensemble standard deviation, while the latter is a local variance decomposition method using the derivative of the output concentration with respect to the (input) rate constants. We preferred the sampling based method for our particular analysis for several reasons. Firstly, the selected method is appropriate for coagulation analysis given that the $10 \%$ to $1000 \%$ range used in it is the expected range of variability of experimentally measured rate constants. Secondly, as shown in Appendix B, although the results from the two procedures will be qualitatively similar, the procedure in [8] yields a more representative statistic.

We simulate clot formation, growth, and lysis in a quiescent pool of plasma exposed to a thrombogenic patch. We then vary the rate constants and analyze the results using the sensitivity analysis procedure in [8]. We report the constants to which the model prediction of fibrin, and of all species' concentrations, are most/least sensitive. We then discuss the results. 


\section{Model Analysis}

The analysis of the model first involves obtaining the solution of the equations at all space points and time instants for each species. The procedure used to solve the PDEs is detailed in subsection 3.1. We then vary the rate constants in a systematic manner, and thereby obtain measures like the ensemble standard deviation and the coefficient of variation of the species concentration. This allows us to calculate the explained variance which measures the sensitivity of the model predictions to the individual rate constants: this procedure is detailed in subsection 3.2.

\subsection{Solution procedure for the model equations}

The 23 equations governing the generation/depletion and diffusion of the various constituents are of the form:

$$
\frac{\partial\left[Y_{k}\right]}{\partial t}=D_{k} \Delta\left[Y_{k}\right]+G_{k} ; k=1, \ldots, 23
$$

Here, $\left[Y_{k}\right]$ denotes the concentration of the constituent $Y_{k}, \frac{\partial\left[Y_{k}\right]}{\partial t}$ denotes the Eulerian time derivative of $\left[Y_{k}\right], G_{k}$ denotes the net rate of generation/depletion of $Y_{k}$ due to enzymatic reactions, while $D_{k}$ denotes the diffusion coefficient of $Y_{k}$. The form for the enzymatic reactions is based on the principles of mass action kinetics. The entire set of 23 equations is listed below: we refer the reader to [2] for the assumptions behind these equations and the references listing the rate constants for each equation.

$$
\begin{aligned}
G_{X I a} & =\frac{k_{11}[I I a][X I]}{K_{11 M}+[X I]}-h_{11}^{A 3}[X I a][A T I I I]-h_{11}^{L 1}[X I a]\left[\alpha_{1} A T\right] \\
G_{X I} & =-\frac{k_{11}[I I a][X I]}{K_{11 M}+[X I]} \\
G_{I X a} & =\frac{k_{9}[X I a][I X]}{K_{9 M}+[I X]}-h_{9}[I X a][A T I I I] \\
G_{I X} & =-\frac{k_{9}[X I a][I X]}{K_{9 M}+[I X]} \\
{[Z] } & =\frac{[V I I I a][I X a]}{K_{d Z}} \\
G_{X a} & =\frac{k_{10}[Z][X]}{K_{10 M}+[X]}-h_{10}[X a][A T I I I]-h_{T F P I}[T F P I][X a] \\
G_{X} & =-\frac{k_{10}[Z][X]}{K_{10 M}+[X]} \\
\left.W_{W}\right] & =\frac{[V a][X a]}{K_{d W}}, \\
G_{I I a} & =\frac{k_{2}[W][I I]}{K_{2 M}+[I I]}-h_{2}[I I a][A T I I I] \\
G_{I I} & =-\frac{k_{2}[W][I I]}{K_{2 M}+[I I]} \\
G_{V I I I a} & =\frac{k_{8}[I I a][V I I I]}{K_{8 M}+[V I I I]}-h_{8}[V I I I a]-\frac{h_{C 8}[A P C][V I I I a]}{H_{C 8 M}+[V I I I a]} \\
G_{V I I I} & =-\frac{k_{8}[I I a][V I I I]}{K_{8 M}+[V I I I]}
\end{aligned}
$$




$$
\begin{aligned}
G_{V a} & =\frac{k_{5}[I I a][V]}{K_{5 M}+[V]}-h_{5}[V a]-\frac{h_{C 5}[A P C][V a]}{H_{C 5 M}+[V a]}, \\
G_{V} & =-\frac{k_{5}[I I a][V]}{K_{5 M}+[V]}, \\
G_{A P C} & =\frac{k_{P C}[I I a][P C]}{K_{P C M}+[P C]}-h_{P C}[A P C]\left[\alpha_{1} A T\right], \\
G_{P C} & =-\frac{k_{P C}[I I a][P C]}{K_{P C M}+[P C]} \\
G_{A T I I I} & =-\left(h_{9}[I X a]+h_{10}[X a]+h_{2}[I I a]+h_{11}^{A 3}[X I a]\right)[A T I I I], \\
G_{T F P I} & =-h_{T F P I}[T F P I][X a] \\
G_{\alpha_{1} A T} & =-h_{P C}[A P C]\left[\alpha_{1} A T\right]-h_{11}^{L 1}[X I a]\left[\alpha_{1} A T\right], \\
G_{I a} & =\frac{k_{1}[I I a][I]}{K_{1 M}+[I]}-\frac{h_{1}[P L A][I a]}{H_{1 M}+[I a]}, \\
G_{I} & =-\frac{k_{1}[I I a][I]}{K_{1 M}+[I]}, \\
G_{t P A} & =0 \\
G_{P L A} & =\frac{k_{P L A}[t P A][P L S]}{K_{P L A M}+[P L S]}-h_{P L A}[P L A]\left[\alpha_{2} A P\right], \\
G_{P L S} & =-\frac{k_{P L A}[t P A][P L S]}{K_{P L A M}+[P L S]} \\
G_{\alpha_{2} A P} & =-h_{P L A}[P L A]\left[\alpha_{2} A P\right] .
\end{aligned}
$$

The equations governing the generation and depletion of the species are formulated based on experimental data for the reaction kinetics. The rate of depletion of a zymogen is equal to the rate of its activation into the corresponding active enzyme. The active enzyme is generated from the zymogen and is depleted by inactivation.

We first non-dimensionalize equation(s) (3.1) using the following formulas: $t^{*}=\frac{t}{T}, x^{*}=\frac{x}{L},\left[Y_{k}^{*}\right]=$ $\frac{\left[Y_{k}\right]}{\left.\left[Y_{k}\right]\right]_{t=0}}, D_{k}^{*}=\frac{D_{k} T}{L^{2}}, G_{k}^{*}=\frac{G_{k} T}{\left.\left[Y_{k}\right]\right|_{t=0}}$.

The non-dimensionalized PDEs to be solved are:

$$
\frac{\partial\left[Y_{k}^{*}\right]}{\partial t^{*}}=D_{k}^{*} \frac{\partial^{2}\left[Y_{k}^{*}\right]}{\partial x^{* 2}}+G_{k}^{*} ; k=1, \ldots, 23 .
$$

We now reduce the PDEs to ODEs by replacing the second order derivative on the right-hand side of equation (3.2) with a $2^{\text {nd }}$ order accurate, three-point central difference scheme given by:

$$
{\frac{\partial^{2}\left[Y_{k}^{*}\right]}{\partial x^{* 2}}}_{i, j}=\frac{\left[Y_{k}^{*}\right]_{i+1, j}-2\left[Y_{k}^{*}\right]_{i, j}+\left[Y_{k}^{*}\right]_{i-1, j}}{\left(\Delta x^{*}\right)^{2}}
$$

Here $j$ refers to the node for the time instant, while $i$ represents the node for the spatial location.

The recast ODEs are given by:

$$
{\frac{d\left[Y_{k}^{*}\right]}{d t^{*}}{ }_{i, j}}=D_{k}^{*} \frac{\left[Y_{k}^{*}\right]_{i+1, j}-2\left[Y_{k}^{*}\right]_{i, j}+\left[Y_{k}^{*}\right]_{i-1, j}}{\left(\Delta x^{*}\right)^{2}}+\left.G_{k}^{*}\right|_{i, j} ; k=1, \ldots, 23 .
$$

The ODEs in equation (3.3) form a system of ODE-IVPs at each spatial node. They are easily solved using a standard Runge-Kutta method of order 4: this procedure yields a solution at each time instant(j), for each spatial location (i) and each species (k).

We use $\Delta x^{*}=0.01$, and $\Delta t^{*}=10^{-5}$. We note that grid independence of the results is checked by solving increasingly small sizes till the reported sensitivity and explained variance values do not change. 
In our case, we fix $\Delta t^{*}$, and decrease $\Delta x^{*}$ till the relative variation in explained variance (equation (3.8)) is acceptably small.

The solution procedure for the equations is as given above; the discretized initial and boundary conditions are implemented as given in Appendix A. The solution procedure and boundary condition implementation are also given in [21].

\subsection{Sensitivity Analysis}

The sensitivity analysis procedure for the model equations is from [8]: we use similar notation for ease of comparison.

We first calculate the standard deviation $\left(\sigma_{k_{j}}^{f}(t)\right.$ : equation (3.4)) which, for a given model species $f$ at a selected time instant $t$, is the variation in concentration of species $f$ due to variation of a specific rate constant $k_{j}$. This standard deviation is calculated for $i$ rate constant variations in a linearly spaced range on a logarithmic (base 10) scale as follows: $i=10 \%, 20 \%, 30 \%, \ldots 100 \%, 200 \%, \ldots 1000 \%$ of the original rate constant value. The ensemble standard deviation for each species $f$ at a given time instant is thus calculated for $i$ variations of a rate constant $k_{j}$. The value in Equation (3.4) is given for a spatially homogeneous system whereas our system of equations models a spatially inhomogeneous system. In order to use the solution of our model equations in equation (3.4), we take the spatial average of species concentrations to be the instantaneous concentration $\left(C_{i}^{f}(t)\right)$ substituted in the equation. $\bar{C}^{f}(t)$ in equation (3.4) denotes the average of the $i$ instantaneous concentrations $\left(C_{i}^{f}(t)\right)$.

$$
\sigma_{k_{j}}^{f}(t)=\left(\frac{\sum_{i=1}^{n}\left(C_{i}^{f}(t)-\bar{C} f(t)\right)^{2}}{n-1}\right)^{\frac{1}{2}}
$$

We then calculate the coefficient of variation $\left(W_{k_{j}}^{f}(t)\right.$ : equation $\left.(3.5)\right)$. This is done by dividing the ensemble standard deviation for species $f$ with the peak value of species $f$ 's concentration obtained using the control model (100\% rate constant value). The concentration of species $f$ obtained using the control model is given by $\hat{C}_{f}(t)$, and its peak value is $\max \left(\hat{C}_{f}(t)\right)$.

$$
W_{k_{j}}^{f}(t)=\frac{\sigma_{k_{j}}^{f}(t)}{\max \left(\hat{C}_{f}(t)\right)} .
$$

Next, we calculate the mean coefficient of variation $\left(\gamma_{k_{j}}(t)\right.$ : equation (3.6)) for each rate constant, by calculating the average of the coefficients of variation for all species $\left(n_{f}=23\right.$, in our case) as given below:

$$
\gamma_{k_{j}}(t)=\frac{\Sigma_{f} W_{k_{j}}^{f}(t)}{n_{f}} .
$$

We then calculate the time-averaged coefficient of variation for each rate constant $\left(\Gamma_{k_{j}}\right.$ : equation (3.7)) by averaging the mean coefficient of variation over the entire time of the simulation (20 minutes in our simulations). Note that the time-averaged coefficient of variation of species concentrations $\left(\Gamma_{k_{j}}\right)$ represents summation over both the number of species $\left(n_{f}=23\right)$ as well as the number of time steps $\left(n_{t}\right)$, and is reported for each rate constant.

$$
\Gamma_{k_{j}}=\frac{\Sigma_{t} \gamma_{k_{j}}(t)}{n_{t}}
$$

The relative importance of the $m^{\text {th }}$ rate constant (among the total of 36) is assessed using the explained variance $\left(E_{v a r}^{m}\right.$ : equation (3.8)) given by:

$$
E_{v a r}^{m}=\frac{\sum_{j=1}^{m} \Gamma_{k_{j}}}{\sum_{j=1}^{36} \Gamma_{k_{j}}}
$$




\section{Results}

We first validate the solution procedure for the equations given in the preceding section by obtaining the results for the control model (100\% rate constant values). We plot fibrin concentration versus (non-dimensional) space and (non-dimensional) time in Figure 2. This solution of the control model is intuitively correct: it shows fibrin concentration increasing up to a particular time, and thereafter decreasing with time due to the onset of fibrinolysis as well as spreading due to diffusion.

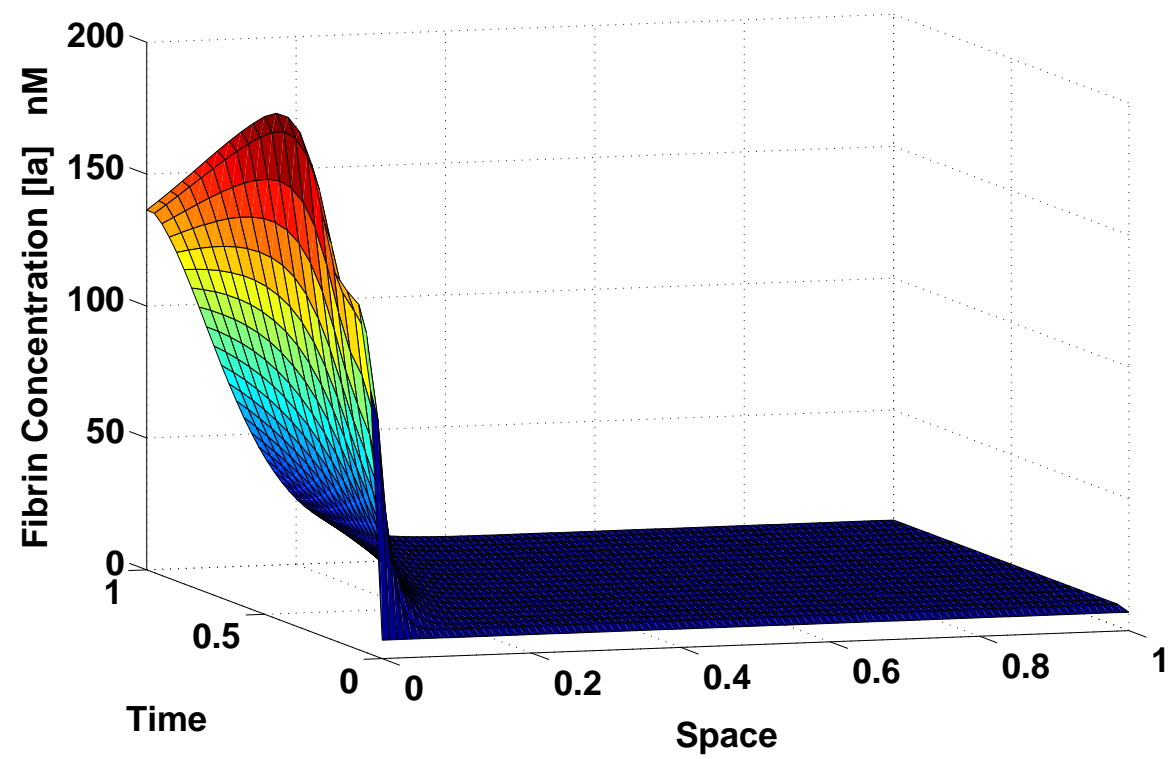

FiguRE 2. Variation of fibrin concentration with space (non-dimensional) and time (nondimensional) for the control model.

Having validated the solution procedure using the above result, we then perform a sensitivity analysis for the model using the procedure detailed earlier. In this we are interested in identifying those rate constants to which the model predictions of fibrin, and of all 23 species taken together, are most/least sensitive. We have established in Appendix B that the sensitivity analysis using the procedure in [17] will yield a similar result to that obtained using the procedure here. We therefore conclude with greater probability that the rate constants identified by our analysis are the ones that affect the model the most/ least.

The time dependent coefficients of variation of fibrin concentration at various time instants (namely $\left.W_{k_{j}}^{I a}(t)\right)$ are displayed in Figure 3. We note that fibrin concentration is most sensitive to the rate constant number 11 that governs activation of prothrombin to thrombin $\left(k_{2}\right)$. It is second most-sensitive to the rate constant number 20 that governs activation of factor $\mathrm{V}$ by thrombin $\left(k_{5}\right)$. Thus, the model predicts that fibrin production is most sensitive to the action of prothrombin, and, prior to that, to the formation of prothrombinase. This prediction corresponds nicely with the experimental observation that fibrin formation is nearly instantaneous once thrombin is formed, and that prothrombinase formation is the most crucial event for the generation of thrombin.

The time dependent mean coefficients of variation (namely $\gamma_{k_{j}}(t)$ ) are displayed in Figure 4 . We find that the rate constant $\left(h_{8}\right)$ governing factor VIIIa inactivation affects model predictions the most, whereas the rate constant $\left(h_{2}\right)$ governing factor IIa (thrombin) association with ATIII affects the model least. 
Summarizing the results in Figure 4, we list the rate constants to which the model is most/least sensitive in Table 3. We see that at several time instants the rate constants $h_{C 8}, H_{C 8 M}$ are the ones that affect the model the most after accounting for the rate constant $h_{8}$. In order to conclude as to which rate constant affects the entire model the most/least, we plot the variation of explained variance $\left(E_{v a r}^{m}\right)$ due to the individual rate constants in Figure 5. The constants are plotted in decreasing order of explained variance. This plot reiterates that the rate constant number 16 ( $h_{8}$ which governs the inactivation of VIIIa) has the highest value of explained variance, and is therefore the constant to which the overall model predictions are most sensitive. Further, the constant numbers $17 \& 18\left(h_{C 8} \& H_{C 8 M}\right.$ which govern the inactivation of VIIIa by APC) have the second highest value of explained variance, so that the model overall is most sensitive to constants governing VIIIa inactivation, whether intrinsic or by APC. We discuss these results in the next section.

\section{Table 3: Time dependent sensitivity of rate constants to uncertainity}

\begin{tabular}{|c|c|c|}
\hline Time (min) & Most sensitive & Least sensitive \\
\hline 2 & $h_{8}, h_{C 8}, H_{C 8 M}, k_{5}, K_{5 M}$ & $h_{2}, K_{P L A M}, H_{1 M}, h_{1}, K_{11 M}$ \\
\hline 4 & $h_{8}, h_{C 8}, H_{C 8 M}, k_{5}, K_{5 M}$ & $h_{2}, K_{11 M}, K_{P L A M}, H_{1 M}, h_{1}$ \\
\hline 6 & $h_{8}, h_{C 8}, H_{C 8 M}, k_{5}, K_{5 M}$ & $h_{2}, K_{11 M}, h_{11}^{L 1}, K_{P L A M}, H_{1 M}$ \\
\hline 8 & $h_{8}, h_{C 8}, H_{C 8 M}, k_{5}, K_{5 M}$ & $h_{2}, K_{11 M}, h_{11}^{L 1}, k_{11}, K_{9 M}$ \\
\hline 10 & $h_{8}, h_{C 8}, H_{C 8 M}, k_{5}, K_{5 M}$ & $h_{2}, K_{11 M}, h_{11}^{L 1}, k_{11}, K_{9 M}$ \\
\hline 12 & $h_{8}, h_{C 8}, k_{5}, H_{C 8 M}, K_{5 M}$ & $h_{2}, K_{11 M}, h_{11}^{L 1}, k_{11}, K_{9 M}$ \\
\hline 15 & $h_{8}, H_{C 8 M}, k_{2}, K_{5 M}, h_{C 8}$ & $h_{2}, K_{11 M}, h_{11}^{L 1}, k_{11}, K_{9 M}$ \\
\hline 20 & $h_{8}, H_{C 8 M}, k_{2}, K_{5 M}, K_{d W}$ & $h_{2}, K_{11 M}, h_{11}^{L 1}, k_{11}, K_{9 M}$ \\
\hline
\end{tabular}

\section{Discussion}

We have performed a sensitivity analysis of the model developed in [2] using the procedure in [8]. We were thus able to identify the constants to which the model was most/least sensitive based on explained variance. Although there was some variation between the results of the model based on explained variance, and that based on (time-dependent) mean coefficient of variation, we consistently found that the rate constant governing the inactivation of factor VIIIa $\left(h_{8}\right)$ caused the maximum change in model predictions, whereas the rate constant governing the association of thrombin with ATIII $\left(h_{2}\right)$ caused the least change. Further, based on explained variance, the model was second most-sensitive to $h_{C 8}$, and third-most sensitive to $H_{C 8 M}$. We therefore conclude that reactions involving the inactivation of factor VIIIa (either intrinsic, or by the action of APC) caused the most change to the model predictions. The sensitivity analysis in [17] will also yield a fundamentally similar result (see Appendix B). We therefore conclude that the rate constants governing VIIIa inactivation, either intrinsic or by activated Protein C, are the ones that greatest affect the predictions of the model in [2].

An identical analysis of the model in [10] shows that the reaction constant that caused the maximum change in their model predictions, after those involving tissue factor (TF), was the intrinsic inactivation of factor VIIIa. In our study, TF is included in the boundary conditions as [TF-VIIa], and a separate analysis of its role is not performed because it appears as a concentration, and not as a rate constant. However, excluding TF, the sensitivity analysis of the model in [2] too gives the result that the rate constant governing inactivation of factor VIIIa affects the model predictions the most. This lends more weight to our analytical result, and makes it a contender for confirmation by means of a separate set of experiments, which should logically follow this study but which we have not performed. These limitations are discussed in the next section. 


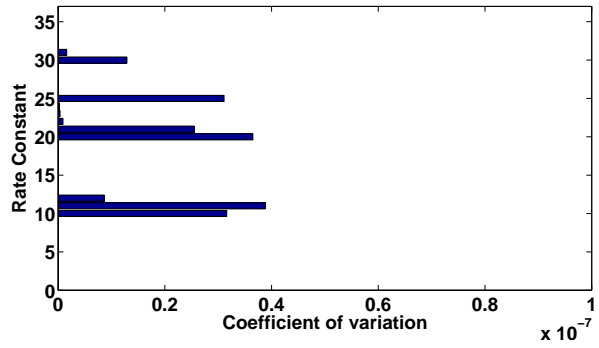

(a) $t=2 \min$

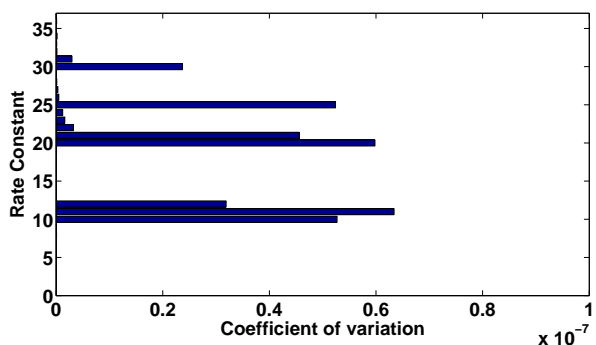

(c) $t=6 \mathrm{~min}$

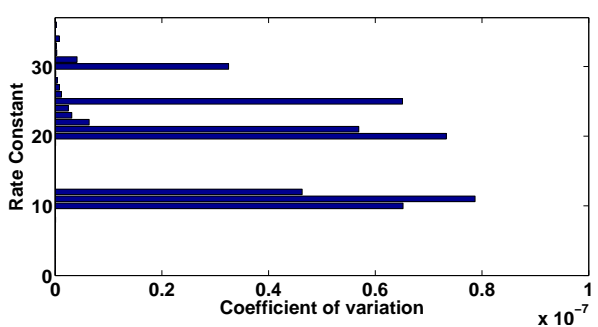

(e) $t=10 \mathrm{~min}$

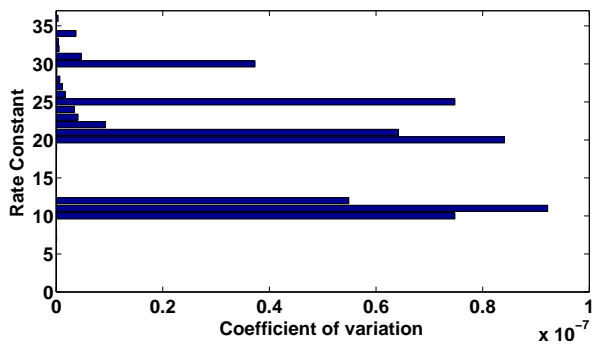

(g) $t=15 \mathrm{~min}$

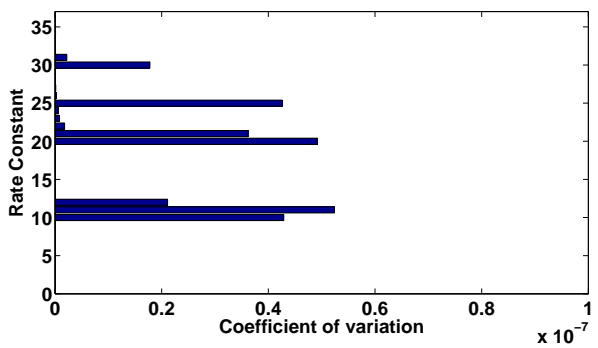

(b) $t=4 \mathrm{~min}$

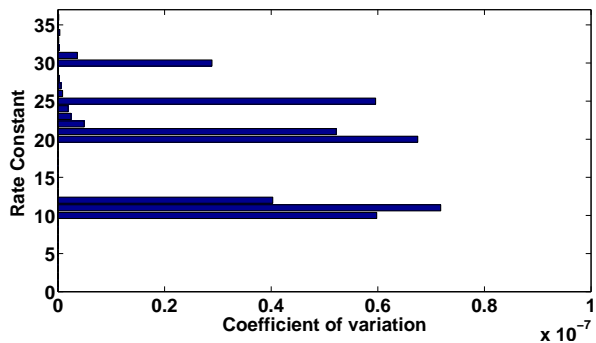

(d) $t=8 \mathrm{~min}$

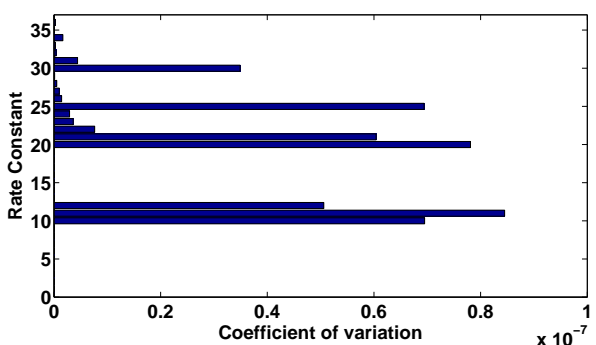

(f) $t=12 \mathrm{~min}$

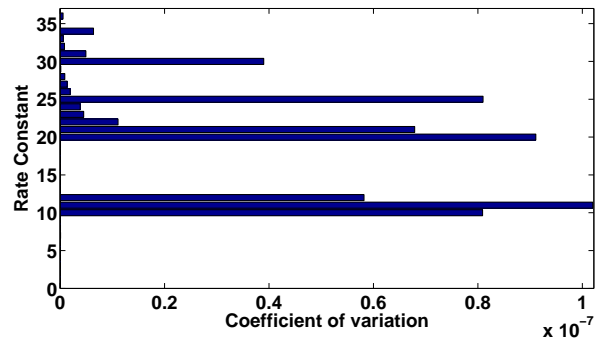

(h) $t=20 \mathrm{~min}$

Figure 3. Time-dependent coefficient of variation of fibrin (Ia) concentration for each rate constant. 


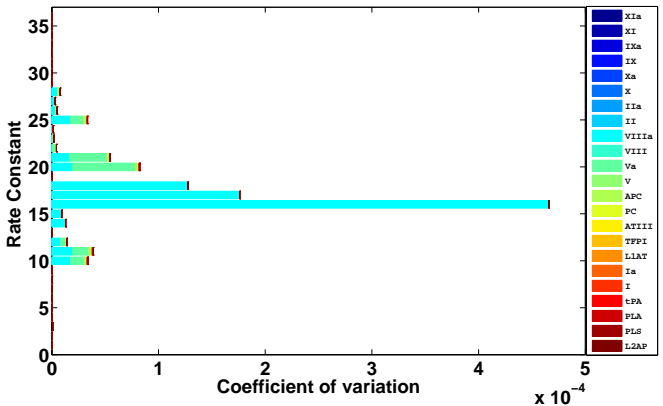

(a) $t=2 \min$

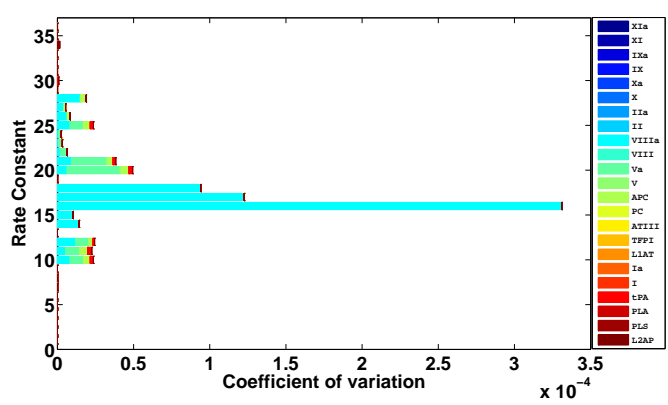

(c) $t=6 \mathrm{~min}$

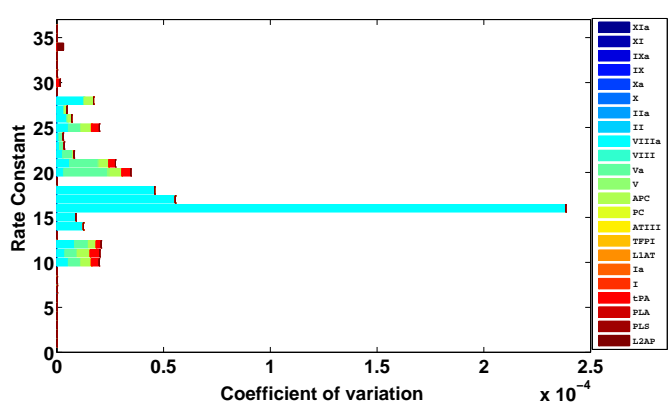

(e) $t=10 \mathrm{~min}$

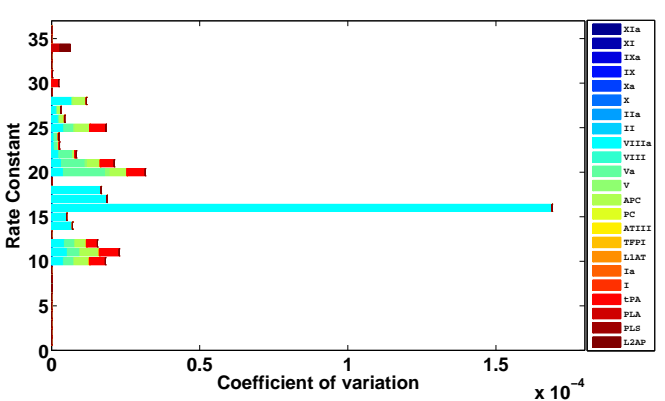

(g) $t=15 \mathrm{~min}$

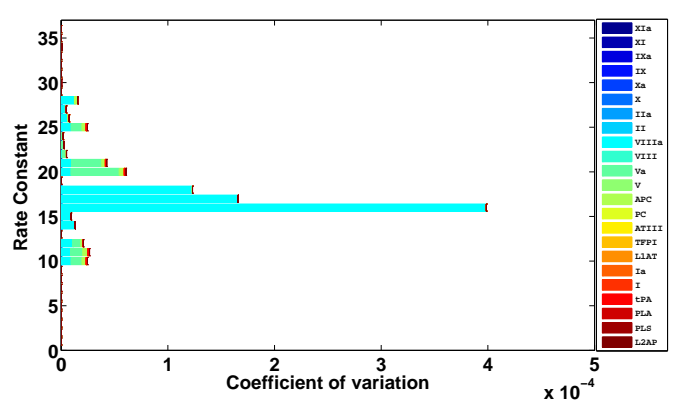

(b) $t=4 \mathrm{~min}$

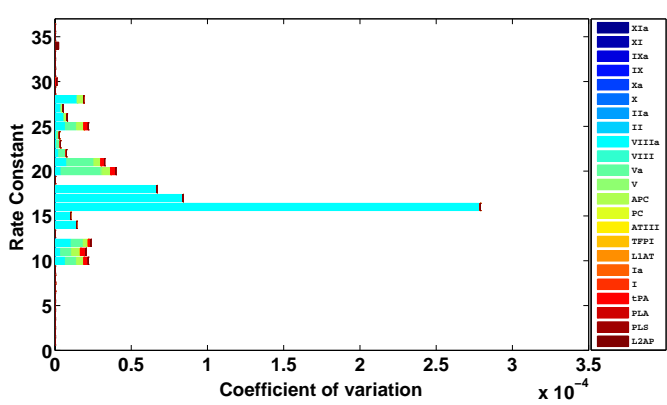

(d) $t=8 \mathrm{~min}$

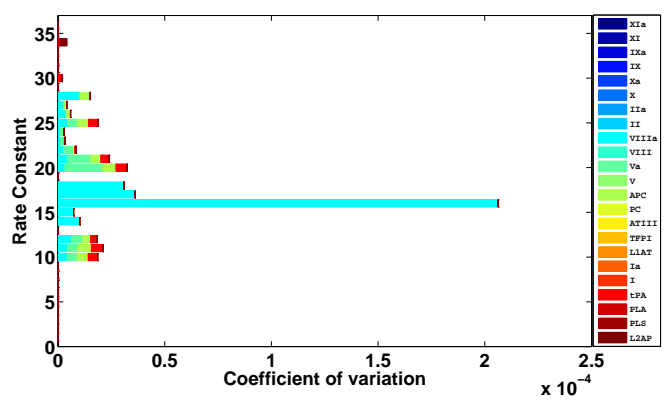

(f) $t=12 \mathrm{~min}$

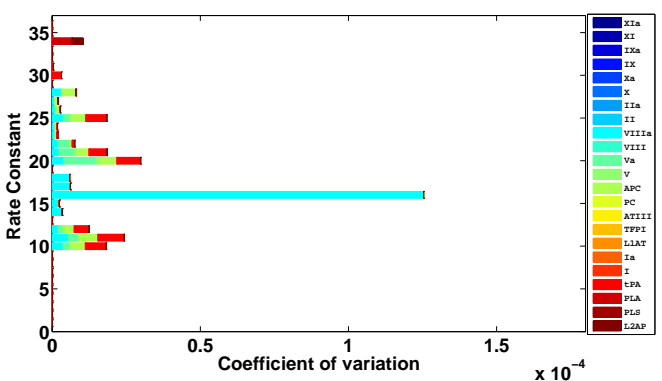

(h) $t=20 \mathrm{~min}$

Figure 4. Time-dependent mean coefficient of variation of all species' concentrations for each rate constant. 


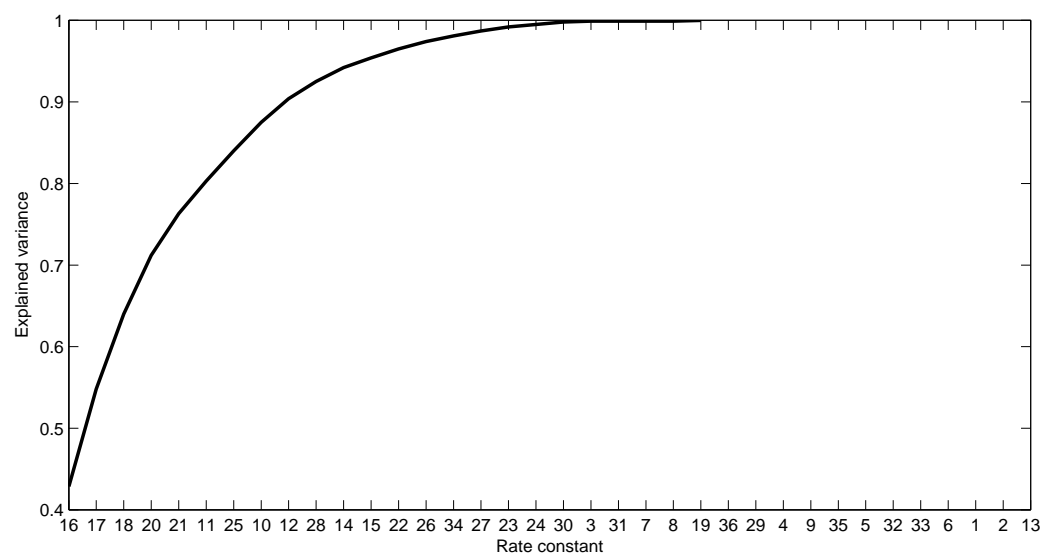

FiguRE 5. Decreasing order of cumulative explained variance $\left(E_{v a r}^{m}\right)$ versus model rate constant number

\subsection{Limitations and Recommendations}

The primary limitation of this study is the lack of experimental data to support the result that VIIIa inactivation most affects clot formation, growth, and lysis: in order to address this limitation, we propose the following experimental design. Platelet poor plasma must be isolated by centrifugation from whole human blood. Tissue factor must be added to initiate coagulation. Concomitantly, activated Protein $\mathrm{C}$ (APC) must be added in stoichiometric excess to deactivate VIIIa and Va, and the effect on fibrin concentration (the end result of coagulation) must be documented. This must be repeated with normal concentrations of, as well as depleted concentrations of, Protein $\mathrm{C}$ in the plasma, and the effect on fibrin concentration must again be documented. This should be followed by tests to record the effects of stoichiometric excess and deficiency of ATIII, TFPI, $\alpha_{1}$ AT ( $\alpha_{1}$-antitrypsin), and PLA (plasmin). The results of this array of tests will pinpoint that inhibitor which causes the greatest change in fibrin concentration. That result should then be compared with the sensitivity analysis prediction that VIIIa inactivation by APC affects model predictions significantly.

Another limitation of this study is that the analysis is done without including flow in the equations. In order to include flow, we need to solve a set of convection-reaction-diffusion equations as advocated in [1]. An extension to include flow would make the study more relevant by studying the sensitivity of the reactions in-vivo. In its present form, the analysis is applicable only to spatially inhomogeneous quiescent conditions in-vitro; even here, given that the diffusion coefficients are all of nearly same magnitude and also that the analysis considers the spatial average of the domain concentration to be the representative concentration at a given time instant, neglecting the diffusion coefficients will not affect the results of the sensitivity analysis. Diffusion is included in our study only for the sake of theoretical completeness.

A key recommendation for a follow-up study is one wherein the concentrations of the most sensitive constituents (in this case [VIIIa] or [VIII]) are varied, and the effect of deficiencies and excesses of these constituents on initiation time, clot size, and concentrations of fibrin is recorded. This will help identify the impact of hematologic malignancies like Hemophilia A or Hemophilia B. Additionally, the results in [8] and [17] point to binding interactions between TF and factors Xa/X/IX/VIIa as being even more critical than those involving VIIIa inactivation. Such results cannot be obtained from the model in [2] because TF is not considered as a separate species. An extension of the model should include factors VII \& VIIa as volumetric species, and include binding interactions between TF on the boundary with $\mathrm{Xa} / \mathrm{X} / \mathrm{IX} / \mathrm{VII}$. The sensitivity analysis must be reperformed on the extended model. 
Acknowledgements. PPN was supported by the Institute Grant for TAs from IIT Hyderabad, and we are grateful for this funding. We thank Dr. C. M. Danforth for explaining the methodology used in [8]. We also thank Dr. M. A. Panteleev for fruitful discussions and insightful comments on the manuscript.

\section{References}

[1] M. Anand, K. Rajagopal, K.R. Rajagopal. A model incorporating some of the mechanical and biochemical factors underlying clot formation and dissolution in flowing blood. J. Theor. Med., 5 (2003), no. 3-4, 183-218.

[2] M. Anand, K. Rajagopal, K.R. Rajagopal. A model for the formation, growth, and lysis of clots in quiescent plasma. A comparison between the effects of antithrombin III deficiency and protein C deficiency. J. Theor. Biol., 253 (2008), no. $4,725-738$.

[3] F.I. Ataullakhanov, V.I. Zarnitsina, A.V. Pokhilko, A.I. Lobanov, O.L. Morozova. Spatio-temporal dynamics of blood coagulation and pattern formation. A theoretical approach, Int. J. Bifurcat. Chaos., 12 (2002), no. 9, $1985-2002$.

[4] N.A. Booth. Fibrinolysis and thrombosis. Baillière Clin. Haem., 12 (1999), no. 3, 423-433.

[5] S.D. Bungay, P.A. Gentry, R.D. Gentry. A mathematical model of lipid-mediated thrombin generation. Math. Med. Biol., 20 (2003), no. 1, 105-129.

[6] S. Butenas, K.G. Mann. Blood coagulation. Biochemistry-Moscow, 67 (2002), no. 1, 3-12.

[7] R.W. Colman, A.W. Clowes, J.N. George, J. Hirsh, V.J. Marder. Overview of Hemostasis, in Hemostasis and Thrombosis. 4th Edition, pp. 1-16, Editors: Colman R. W., Hirsh J., Marder V. J., Clowes A. W., and George J. N., Lippincott, Williams and Wilkins, 2001.

[8] C.M. Danforth, T. Orfeo, K.G. Mann, K.E. Brummel-Ziedins, S.J. Everse. The impact of uncertainty in a blood coagulation model. Math. Med. Biol., 26 (2009), no. 4, 323-336.

[9] B. Furie, B.C. Furie. Molecular basis of blood coagulation, in Hematology : Basic principles and practice. 3rd Edition, 1783-1804, Editors: Hoffman R., Benz E. J., Shattil S. J., Furie B., Cohen H. J., Silberstein L. E., and McGlave P., Churchill Livingstone, 2000.

[10] M.F. Hockin, K.C. Jones, S.J. Everse, K.G. Mann. A model for the stoichiometric regulation of blood coagulation. J. Biol. Chem., 277 (2002), no. 21, 18322-18333.

[11] M. Kalafatis, J.O. Egan, C. vant Veer, K.M. Cawthern, K.G. Mann. The regulation of clotting factors. Crit. Rev. Eukar. Gene, 7 (1997), no. 3, 241-280.

[12] A.L. Karsan, J.M. Harlan. The blood vessel wall, in Hematology : Basic principles and practice. 3rd Edition, 17701782, Editors: Hoffman R., Benz E. J., Shattil S. J., Furie B., Cohen H. J., Silberstein L. E., and McGlave P., Churchill Livingstone, 2000.

[13] A.L. Kuharsky, A.F. Fogelson. Surface mediated control of blood coagulation: the role of binding site densities and platelet deposition. Biophys. J., 80 (2001), no. 3, 1050-1094.

[14] D.E. Lacroix, M. Anand. A model for the formation, growth, and dissolution of clots in vitro. Effect of the intrinsic pathway on antithrombin III deficiency and protein C deficiency. Int. J. Adv. Eng. Sci. Appl. Math., 3 (2012), no. 1-4, 93-105.

[15] S.N. Levine. Enzyme amplifier kinetics. Science, 152 (1966), no. 3722, 651-653.

[16] H.R. Lijnen, D. Collen. Molecular and cellular basis of fibrinolysis, in Hematology : Basic principles and practice , 3rd Edition, 1804-1814, Editors: Hoffman R., Benz E. J., Shattil S. J., Furie B., Cohen H. J., Silberstein L. E., and McGlave P., Churchill Livingstone, 2000.

[17] D. Luan, M. Zai, J.D. Varner. Computationally derived points of fragility of a human cascase are consistent with current therapeutic strategies. PLOS Comput. Biol., 3 (2007), no. 7, e142.

[18] K.G. Mann, D. Gaffney, E.G. Bovill. Molecular biology, biochemistry, and lifespan of plasma coagulation factors. in Williams Hematology, 5th Edition, 1205-1226, Editors: Beutler E., Lichtman M., Coller B. S., and Kipps T. J., McGraw Hill Inc., 1995.

[19] K.G. Mann, K. Brummel-Ziedins, T. Orfeo, S. Butenas. Models of blood coagulation. Blood Cell. Mol. Dis., 36 (2006), 108-117.

[20] T. Orfeo, S. Butenas, K.E. Brummel-Ziedins, K.G. Mann. The tissue factor requirement in blood coagulation. J. Biol. Chem., 280 (2005), no. 52, 42887-42896.

[21] P.N. Paluri. Sensitivity analysis of a mathematical model for blood coagulation and fibrinolysis. Master's thesis, Indian Institute of Technology Hyderabad, Yeddumailaram, AP, INDIA, 2012.

[22] M.A. Panteleev, M.V. Ovanesov, D.A. Kireev, A.M. Shibeko, E.I. Sinauridze, N.M. Ananyeva, A.A. Butylin, E.L. Saenko, F.I. Ataullakhanov. Spatial propagation and localization of blood coagulation are regulated by intrinsic and protein C pathways, respectively Biophys. J., 90 (2006), no. 5, 1489-1500.

[23] A. Sequeira, R.F. Santos, T. Bodnar. Blood Coagulation Dynamics: Mathematical modeling and stability results. Math. Biosci. Eng., 8 (2011), no. 2, 425-443.

[24] Wells K. R., Blood Coagulation, http://health.yahoo.net/galecontent/blood-coagulation/2 Accessed May $10^{t h} 2012$. 


\section{A. Initial and Boundary conditions for the model equations}

The initial conditions for the constituents are obtained by selecting the initial concentrations of fibrinogen(I), zymogens (II, V, VIII, IX, X, XI, PLS), inhibitors (ATIII, TFPI, $\alpha_{1} \mathrm{AT}, \alpha_{2} \mathrm{AP}$ ), Protein C (PC), and tPA to be their physiologic values obtained from the literature, and assuming $0.1 \%$ initial activation for fibrin (Ia), enzymes (IIa, Va, VIIIa, IXa, Xa, XIa, PLA), and active Protein C (APC). The values are listed in Table 4 (these are identical to those in [2]).

Table 4: Initial conditions.

\begin{tabular}{|c|c|c|}
\hline Species & Initial concentration (nM) & Reference \\
\hline \hline I & 7000 & {$[18]$} \\
\hline Ia & 7.0 & {$[18]$} \\
\hline II & 1400 & {$[18]$} \\
\hline IIa & 1.4 & \\
\hline V & 20 & {$[18]$} \\
\hline Va & 0.02 & \\
\hline VIII & 0.7 & {$[18]$} \\
\hline VIIIa & 0.0007 & {$[18]$} \\
\hline IX & 90 & \\
\hline IXa & 0.09 & {$[5]$} \\
\hline X & 170 & \\
\hline Xa & 0.17 & {$[11]$} \\
\hline XI & 30 & {$[18]$} \\
\hline XIa & 0.03 & {$[18]$} \\
\hline ATIII & 3400 & \\
\hline TFPI & 2.5 & {$[7]$} \\
\hline PC & 60 & {$[4]$} \\
\hline APC & 0.06 & {$[16]$} \\
\hline$\alpha_{1}$ AT & 45000 & {$[7]$} \\
\hline tPA & 0.08 & \\
\hline PLS & 2180 & \\
\hline PLA & 2.18 & \\
\hline$\alpha_{2}$ AP & 105 & \\
\hline
\end{tabular}

The problem we simulate is that of clot formation, growth, and lysis in a quiescent pool of plasma exposed to a thrombogenic patch. The further end of the domain $\left(x^{*}=1 \equiv i=I\right)$ is the layer of plasma exposed to the air, and this layer is impermeable to the movement of all constituents. The corresponding boundary condition is:

$$
\left.\frac{\partial\left[Y_{k}^{*}\right]}{\partial x^{*}}\right|_{x^{*}=1, j}=0, k=1, \ldots, 23 .
$$

A $2^{\text {nd }}$-order accurate, three-point, one-sided difference scheme results in the following equation:

$$
\frac{-\left[Y_{k}^{*}\right]_{I-2, j}+4\left[Y_{k}^{*}\right]_{I-1, j}-3\left[Y_{k}^{*}\right]_{I, j}}{\Delta x^{*}}=0 \forall k .
$$

Equation (A.1) is used to solve for the value of $\left[Y_{k}^{*}\right]_{I, j}$ once $\left[Y_{k}^{*}\right]_{I-1, j}$ and $\left[Y_{k}^{*}\right]_{I-2, j}$ are obtained using the RK4 method for equation (3.3).

The near end of the domain $\left(x^{*}=0 \equiv i=1\right)$ denotes the thrombogenic plane. The boundary conditions at this node are:

$$
\left.\frac{\partial\left[Y_{k}^{*}\right]}{\partial x^{*}}\right|_{x^{*}=0, j}=0 \forall k \text { except }(I X a, I X, X a, X, t P A) .
$$


For 18 constituents (full 23 after excluding IXa, IX, Xa, X, tPA), we use a $2^{\text {nd }}$-order accurate, threepoint, one-sided finite difference scheme as below:

$$
\frac{-\left[Y_{k}^{*}\right]_{3, j}+4\left[Y_{k}^{*}\right]_{2, j}-3\left[Y_{k}^{*}\right]_{1, j}}{\Delta x^{*}}=0 .
$$

Equation (A.2) is used to solve for the value of $\left[Y_{k}^{*}\right]_{1, j}$ once $\left[Y_{k}^{*}\right]_{2, j}$ and $\left[Y_{k}^{*}\right]_{3, j}$ are obtained using the RK-4 method in equation (3.3).

For the 5 constituents- IXa, IX, Xa, X, tPA- the boundary conditions at this node $\left(x^{*}=0 \equiv i=1\right)$ are:

$$
\begin{gathered}
\left.\frac{\partial[I X a]}{\partial x}\right|_{x=0}=-\frac{k_{7,9}[I X][T F-V I I a]}{K_{7,9 M}+[I X]} \frac{L}{D_{I X a}}=-\left.\frac{D_{I X}}{D_{I X a}} \frac{\partial[I X]}{\partial x}\right|_{x=0}, \\
\left.\frac{\partial[X a]}{\partial x}\right|_{x=0}=-\frac{k_{7,10}[X][T F-V I I a]}{K_{7,10 M}+[X]} \frac{L}{D_{X a}}=-\left.\frac{D_{X}}{D_{X a}} \frac{\partial[X]}{\partial x}\right|_{x=0}, \\
\left.\frac{\partial[t P A]}{\partial x}\right|_{x=0}=-\left(k_{t P A}^{C}+\left.k_{t P A}^{I I a}[I I a]\right|_{x=0}+\left.k_{t P A}^{I a}[I a]\right|_{x=0}\right)[E N D O] \frac{L}{D_{t P A}} .
\end{gathered}
$$

After non-dimensionalizing the above equations and rearranging them, a quadratic equation is obtained for the constituents: IXa, IX, Xa, and X. The boundary concentrations of each of these constituents is obtained after solving for the corresponding $\left[Y_{k}^{*}\right]_{2, j}$ and $\left[Y_{k}^{*}\right]_{3, j}$ using the RK4 method in equation (3.3).

The boundary concentrations for IX, IXa are given by:

$$
\begin{aligned}
a= & 1, \\
b= & \frac{K_{7,9 M}}{\left.[I X]\right|_{t=0}}-\frac{1}{3}\left(4\left[I X^{*}\right]_{2, j}-\left[I X^{*}\right]_{3, j}\right)+\left(\frac{L^{2} \Delta x^{*} k_{7,9}}{3\left(\left.[I X]\right|_{t=0}\right) D_{I X}}\right)[\text { TF }- \text { VII }], \\
c= & -\frac{K_{7,9 M}}{3\left(\left.[I X]\right|_{t=0}\right)}\left(4\left[I X^{*}\right]_{2, j}-\left[I X^{*}\right]_{3, j}\right), \\
I X_{1, j}^{*}= & \frac{-b+\sqrt{b^{2}-4 a c}}{2 a} ; \\
I X a_{1, j}^{*}= & \frac{\left(4\left[I X a^{*}\right]_{2, j}-\left[I X a^{*}\right]_{3, j}\right)}{3}+ \\
& \frac{L^{2} \Delta x^{*} k_{7,9}\left[I X^{*}\right]_{1, j}\left(\left.[I X]\right|_{t=0}\right)}{\left(\left.[I X a]\right|_{t=0}\right) D_{I X a}} \frac{[T F-V I I a]}{K_{7,9 M}+\left[I X^{*}\right]_{1, j}\left(\left.[I X]\right|_{t=0}\right)} .
\end{aligned}
$$

The values of the kinetic constants $k_{7,9}, K_{7,9 M}$ are given in [2]. The procedure to estimate the surface concentration, [TF-VIIa], is also given therein. Briefly, the procedure involves generating the concentration curve for total [TF-VIIa] by scaling experimental data available for total [TF-VIIa] when 5 pM of $\mathrm{TF}$ is introduced into synthetic plasma.

The boundary concentrations for $\mathrm{X}$ and $\mathrm{Xa}$ are given by:

$$
\begin{aligned}
d= & 1 \\
e= & \frac{K_{7,10 M}}{\left.[X]\right|_{t=0}}-\frac{1}{3}\left(4\left[X^{*}\right]_{2, j}-\left[X^{*}\right]_{3, j}\right)+\left(\frac{L^{2} \Delta x^{*} k_{7,10}}{3\left(\left.[X]\right|_{t=0}\right) D_{X}}\right)[T F-\text { VII }], \\
f= & -\frac{K_{7,10 M}}{3\left(\left.[X]\right|_{t=0}\right)}\left(4\left[X^{*}\right]_{2, j}-\left[X^{*}\right]_{3, j}\right) \\
X_{1, j}^{*}= & \frac{-e+\sqrt{e^{2}-4 d f}}{2 d} ; \\
X a_{1, j}^{*}= & \frac{\left(4\left[X a^{*}\right]_{2, j}-\left[X a^{*}\right]_{3, j}\right)}{3}+ \\
& \frac{L^{2} \Delta x^{*} k_{7,10}\left[X^{*}\right]_{1, j}\left(\left.[X]\right|_{t=0}\right)}{\left(\left.[X a]\right|_{t=0}\right) D_{X a}} \frac{[T F-V I I a]}{K_{7,10 M}+\left[X^{*}\right]_{1, j}\left(\left.[X]\right|_{t=0}\right)} .
\end{aligned}
$$


The values of the kinetic constants $k_{7,10}, K_{7,10 M}$ are given in [2].

The boundary concentration for tPA is given by:

$$
\begin{aligned}
F_{t P A}^{C} & =6.52 \times 10^{-13} n M m^{2} \min ^{-1} \\
F_{t P A}^{I a} & =5.059 \times\left. 10^{-18}\left[\text { Ia }^{*}\right]\right|_{x=0}[I a]_{t=0} n M m^{2} \min ^{-1}, \\
F_{t P A}^{I I a} & =9.27 \times\left. 10^{-12} e^{-134.8\left(t-T_{0}\right)}\left[I^{*} a^{*}\right]\right|_{x=0}[I I a]_{t=0} n M m^{2} \min ^{-1}, \\
F_{t P A} & =F_{t P A}^{C}+F_{t P A}^{I a}+F_{t P A}^{I I a}, \\
t P A_{1, j}^{*} & =\frac{\left(4\left[t P A^{*}\right]_{2, j}-\left[t P A^{*}\right]_{3, j}\right)}{3}+\frac{L^{2} \Delta x^{*}[E N D O] F_{t P A}}{3\left(\left.[t P A]\right|_{t=0}\right) D_{t P A}} .
\end{aligned}
$$

Here $T_{0}$ is the instant at which $\left.\left[I I a^{*}\right]\right|_{x=0}$ is non zero. The surface concentration of endothelial cells $\left([E N D O]=2.0 \times 10^{9}\right.$ cells $\left./ \mathrm{m}^{2}\right)$ is picked to be an approximation of physiologic surface concentration of endothelial cells in the human vasculature [12].

\section{B. Comparison between the sensitivity coefficients obtained using [17] and [8]}

The Overall State Sensitivity Coefficient (OSSC) of the species concentrations with respect to variation in rate constant $k_{j}$ is the measure of sensitivity used in [17]. It is given by the equation:

$$
S_{0 j}(t)=\frac{p_{j}^{*}}{N}\left(\Sigma_{k=1}^{N_{T}} \Sigma_{i=1}^{N}\left[\frac{1}{x_{i}^{*}} \frac{\partial x_{i}}{\partial p_{j}}\right]_{t_{k}}^{2}\right)^{1 / 2} .
$$

In the above equation, $N_{T}$ represents the number of time instants, while $N$ represents the number of proteins in the model. Further, $\frac{\partial x_{i}}{\partial p_{j}}$ represents the instantaneous change (at time $t_{k}$ ) of concentration of species $i$ due to the variation in rate constant $p_{j} . x_{i}^{*}$ represents the unperturbed concentration of species $i$, while $p_{j}^{*}$ represents the unperturbed rate constant $p_{j}$.

The procedure in [8] results in a time-averaged coefficient of variation that is obtained by combining equations (3.4), (3.5), (3.6) \& (3.7). This coefficient is given by the equation below:

$$
\Gamma_{k_{j}}=\frac{1}{n_{t}} \frac{1}{n_{f}}\left(\Sigma_{t=0}^{n_{t}} \Sigma_{f=1}^{n_{f}} \frac{1}{\max \left(\hat{C}_{f}(t)\right)}\left[\frac{\Sigma_{i=1}^{n}\left(C_{i}^{f}(t)-\bar{C}^{f}(t)\right)^{2}}{n-1}\right]^{\frac{1}{2}}\right)
$$

Rewriting equation (B.1) using the notation in [8], we get:

$$
S_{0 j}(t)=\frac{k_{j}^{*}}{n_{f}}\left(\Sigma_{t=1}^{n_{t}} \Sigma_{f=1}^{n_{f}}\left[\frac{1}{C^{f *}(t)} \frac{\partial C^{f}(t)}{\partial k_{j}}\right]_{t_{t}}^{2}\right)^{1 / 2} .
$$

Here, comparing equation (B.3) with equation (B.2), we note that [17] uses $C^{f *}(t)$ instead of $\max \left(\hat{C}_{f}(t)\right)$, uses $\left[\frac{\partial C^{f}(t)}{\partial k_{j}}\right]_{t_{t}}^{2}$ instead of $\left[\frac{\sum_{i=1}^{n}\left(C_{i}^{f}(t)-\overline{C^{f}}(t)\right)^{2}}{n-1}\right]$, and includes the term $\frac{k_{j}^{*}}{n_{t}}$ that is not present in Equation B.2. The implications of these differences are now discussed.

Using $C^{f *}(t)$ instead of $\max \left(\hat{C}_{f}(t)\right)$ results only in a difference of a multiplicative factor, and will make no (other) difference in the value reported by the OSSC and $\Gamma_{k_{j}} \cdot\left[\frac{\partial C^{f}(t)}{\partial k_{j}}\right]_{t_{t}}^{2}$ in equation B.3 is obtained by perturbing the rate constants randomly within a finite range of $\pm 50 \%$. The value of $\left[\frac{\partial C^{f}(t)}{\partial k_{j}}\right]_{t_{t}}^{2}$ is thus obtained only from two (random) variations within a narrow range of $50 \%$ to $150 \%$. The implementation of the term $\left[\frac{\partial C^{f}(t)}{\partial k_{j}}\right]_{t_{t}}^{2}$ in [17] is only by means of numerical differences. On the other hand, the term $\left[\frac{\Sigma_{i=1}^{n}\left(C_{i}^{f}(t)-\bar{C} f(t)\right)^{2}}{n-1}\right]$ in $[8]$ is calculated as the average of nineteen rate constant variations within a $10 \%$ to $1000 \%$ range. Given the similarities in implementation, the results from the latter term will yield 
a more representative measure of the effect of rate constant variation. Finally, multiplication by $\frac{k_{j}^{*}}{n_{t}}$ to calculate the OSSC will report values that are different from each other by the same factor as the original rate constants. Given the comparisons of the different terms, we conclude that, although there are differences in the values, the two measures $\left(S_{0 j}(t)\right.$, and $\left.\Gamma_{k_{j}}\right)$ in equations (B.1) and (B.2) are qualitatively similar. Further, a more representative value is obtained using the method of [8]. 